\title{
No-go theorem for spatially regular boson stars made of static nonminimally coupled massive scalar fields
}

\author{
Shahar Hod ${ }^{1,2, a}$ \\ ${ }^{1}$ The Ruppin Academic Center, 40250 Emeq Hefer, Israel \\ 2 The Hadassah Academic College, 91010 Jerusalem, Israel
}

Received: 25 November 2018 / Accepted: 28 December 2018 / Published online: 16 January 2019

(C) The Author(s) 2019

\begin{abstract}
We present a compact theorem which reveals the fact that static spatially regular massive scalar fields with nonminimal coupling to gravity cannot form spherically symmetric asymptotically flat horizonless matter configurations. In particular, the no-go theorem rules out the existence of boson stars made of static scalar fields with generic values of the physical parameter $\xi$ which quantifies the coupling between the spacetime curvature and the massive bosonic fields.
\end{abstract}

\section{Introduction}

The non-linearly coupled Einstein-massive-scalar field equations have a physically interesting and mathematically elegant structure that has attracted the attention of researches during the last five decades (see [1-44] and references therein). In particular, this physical system is known to possess asymptotically flat bound-state solutions in the form of rotating hairy black holes that support spatially regular stationary massive scalar field configurations [15-44].

Intriguingly, the phenomenologically rich Einsteinmassive-scalar system also admits horizonless bound-state solutions known as boson stars. These physically interesting compact objects are made of spatially regular stationary massive scalar fields. The physical and mathematical properties of these self-gravitating horizonless matter configurations have been extensively explored by physicists and mathematicians (see [1,2] and references therein). In particular, the intriguing possibility has been considered in the physics literature that boson stars may contribute to the elusive dark matter distributions in galaxies [1,2]. In addition, compact bound-state scalar configurations (boson stars) have been considered in the literature as physically exotic horizonless mimickers of classical black-hole spacetimes [1,2].

\footnotetext{
a e-mail: shaharhod@gmail.com
}

The main goal of the present paper is to reveal the intriguing fact that, as opposed to stationary matter fields, static selfgravitating massive scalar fields with non-minimal coupling to gravity cannot form spatially regular horizonless boundstate matter configurations (boson stars). In this context, it is interesting to note that the asymptotic analysis of the nonlinearly coupled Einstein-scalar field equations presented in [45] can be used to rule out the existence of static boson stars (spatially regular matter configurations) made of nonminimally coupled massive scalar fields in the dimensionless regime $\xi<0$ of the nonminimal coupling parameter ${ }^{1}$.

In the present paper we shall explore the physical and mathematical properties of the Einstein-scalar equations in the complementary physical regime $\xi \geq 0$ of the dimensionless nonminimal coupling parameter. In particular, below we shall present a remarkably compact no-go theorem for static boson stars which reveals the fact that the non-linearly coupled Einstein-massive-scalar field equations do not admit static spatially regular bound-state solutions made of selfgravitating scalar fields with nonminimal coupling to gravity.

\section{Description of the system}

We consider a spatially regular asymptotically flat spacetime whose curvature is sourced by a static self-gravitating massive scalar field $\psi$ with nonminimal coupling to gravity. The curved spherically symmetric spacetime is described by the line element $[9]^{2,3}$

$d s^{2}=-e^{v} d t^{2}+e^{\lambda} d r^{2}+r^{2}\left(d \theta^{2}+\sin ^{2} \theta d \phi^{2}\right)$,

\footnotetext{
${ }^{1}$ The dimensionless physical parameter $\xi$ [see Eq. (5) below] quantifies the strength of coupling between the bosonic matter fields and the characteristic curvature parameter of the spacetime.

2 We use the familiar Schwarzschild spacetime coordinates $(t, r, \theta, \phi)$.

${ }^{3}$ Natural units in which $G=c=\hbar=1$ are used throughout the paper.
} 
where $v=v(r)$ and $\lambda=\lambda(r)$.

The metric functions describing the spatially regular matter configurations are characterized by the near-origin functional behaviors [46-48]

$e^{\lambda}(r \rightarrow 0)=1+O\left(r^{2}\right) ; 0<e^{\nu}<\infty$

with $^{4}$

$\lambda^{\prime}(r \rightarrow 0) \rightarrow 0 ; v^{\prime}(r \rightarrow 0) \rightarrow 0$.

In addition, the metric functions of the asymptotically flat spacetime are characterized by the far-region $(r \rightarrow \infty)$ functional relations [9]

$v(M / r \rightarrow 0) \sim M / r ; \lambda(M / r \rightarrow 0) \sim M / r$,

where $M$ is the asymptotically measured finite ADM mass of the spatially regular static matter configuration.

The self-gravitating scalar field is characterized by the action $[9]^{5}$

$S=S_{E H}-\frac{1}{2} \int\left(\partial_{\alpha} \psi \partial^{\alpha} \psi+\mu^{2} \psi^{2}+\xi R \psi^{2}\right) \sqrt{-g} d^{4} x$,

where $\mu^{6}$ is the mass of the matter field and the dimensionless nonminimal coupling parameter $\xi$ quantifies the coupling between the scalar curvature $R(r)$ [see Eq. (22) below] of the spatially regular spacetime and the massive scalar field $\psi$. For later purposes we note that asymptotically flat spacetimes are characterized by the far-region functional behavior [9]

$R(M / r \rightarrow 0) \rightarrow 0$

of the scalar curvature. In addition, spatially regular nonminimally coupled scalar matter configurations are characterized by asymptotically finite radial eigenfunctions. In particular, as discussed in [9], the bounded functional relation

$-\infty<8 \pi \xi \psi^{2}<1$ for $M / r \ll 1$.

for the scalar eigenfunction guarantees that the corresponding physically acceptable curved spacetimes are characterized by finite and positive values of the asymptotic effective gravitational constant $G_{\text {eff }}=G\left(1-8 \pi G \xi \psi^{2}\right)$ [9].

The spatial and temporal functional behaviors of the nonminimally coupled massive scalar fields with the action (5) are governed by the compact partial differential equation [9]

$\partial_{\alpha} \partial^{\alpha} \psi-\left(\mu^{2}+\xi R\right) \psi=0$.

\footnotetext{
${ }^{4}$ We use the prime symbol ' to denote a radial derivative with respect to the areal coordinate $r$.

${ }^{5}$ Here $S_{\mathrm{EH}}$ is the Einstein-Hilbert action of general relativity.

${ }^{6}$ Note that the physical parameter $\mu$ in the action (5) stands for $\mu / \hbar$. This mass parameter of the nonminimally coupled scalar field therefore has the dimensions of (length) ${ }^{-1}$.
}

In particular, for spherically symmetric static matter configurations, one obtains from (1) and (8) the characteristic ordinary differential equation

$\psi^{\prime \prime}+\frac{1}{2}\left(\frac{4}{r}+v^{\prime}-\lambda^{\prime}\right) \psi^{\prime}-\left(\mu^{2}+\xi R\right) e^{\lambda} \psi=0$,

which determines the spatial behavior of the scalar eigenfunction in the curved spacetime (1).

The components of the energy-momentum tensor $T_{\beta}^{\alpha}$, which characterizes the nonminimally coupled static scalar fields with the action (5), are given by the functional expressions [9]

$$
\begin{aligned}
T_{t}^{t}= & e^{-\lambda} \frac{\xi\left(4 / r-\lambda^{\prime}\right) \psi \psi^{\prime}+(2 \xi-1 / 2)\left(\psi^{\prime}\right)^{2}+2 \xi \psi \psi^{\prime \prime}}{1-8 \pi \xi \psi^{2}} \\
& -\frac{\mu^{2} \psi^{2}}{2\left(1-8 \pi \xi \psi^{2}\right)}, \\
T_{r}^{r}= & e^{-\lambda \frac{\xi\left(4 / r+v^{\prime}\right) \psi \psi^{\prime}+\left(\psi^{\prime}\right)^{2} / 2}{1-8 \pi \xi \psi^{2}}} \\
& -\frac{\mu^{2} \psi^{2}}{2\left(1-8 \pi \xi \psi^{2}\right)}
\end{aligned}
$$

and

$$
\begin{aligned}
& T_{\theta}^{\theta}=T_{\phi}^{\phi} \\
& =e^{-\lambda} \frac{\xi\left(2 / r+v^{\prime}-\lambda^{\prime}\right) \psi \psi^{\prime}+(2 \xi-1 / 2)\left(\psi^{\prime}\right)^{2}+2 \xi \psi \psi^{\prime \prime}}{1-8 \pi \xi \psi^{2}} \\
& -\frac{\mu^{2} \psi^{2}}{2\left(1-8 \pi \xi \psi^{2}\right)} .
\end{aligned}
$$

As explicitly proved in [9], physically acceptable spacetimes are characterized by finite components of the energymomentum tensor:

$\left\{\left|T_{t}^{t}\right|,\left|T_{r}^{r}\right|,\left|T_{\theta}^{\theta}\right|,\left|T_{\phi}^{\phi}\right|\right\}<\infty$.

\section{The no-go theorem for the static nonminimally coupled massive scalar field configurations (boson stars) in the regime $\xi \geq 0$}

As emphasized above, the asymptotic $(r \gg M)$ analysis of the Einstein-scalar field equations presented in $[45]^{7}$ rules out the existence of static boson stars made of self-gravitating massive scalar fields in the dimensionless regime $\xi<0$ of the nonminimal coupling parameter. In the present section we shall complete the no-go theorem for the static sector

\footnotetext{
${ }^{7}$ It is interesting to note that the no-go theorem presented in [13], which is based on the asymptotic large- $r$ functional behavior of the nonminimally coupled massive scalar field configurations, is valid for generic inner boundary conditions. In particular, the no-go theorem of [13] is valid for both horizonless spacetimes describing boson stars with a spatially regular origin and for black-hole spacetimes which are characterized by inner absorbing boundary conditions (event horizons) [13].
} 
of horizonless boson stars. In particular, we shall explicitly prove that nonminimally coupled massive scalar fields with a dimensionless field-curvature coupling parameter in the physical regime $\xi \geq 0$ cannot form spatially regular asymptotically flat static matter configurations.

To this end, we first note that the radial scalar equation (9) takes the simple asymptotic form [see Eqs. (4) and (6)] [45]

$\psi^{\prime \prime}+\frac{2}{r} \psi^{\prime}-\mu^{2} \psi=0$

in the $M / r \ll 1$ region. The physically acceptable (normalizable) radial solution of (14) which respects the bounded asymptotic behavior (7) of physically acceptable spacetimes [9] is given by

$\psi(r)=A \cdot \frac{e^{-\mu r}}{\mu r}$ for $M / r \ll 1$,

where $A$ is a normalization constant.

We shall now prove that the eigenfunction $\psi(r)$, which characterizes the spatial behavior of the spherically symmetric static scalar field configurations, has a non-monotonic dependence on the areal coordinate $r$. In particular, if the radial scalar eigenfunction is characterized by the near-origin relation $\psi(r \rightarrow 0) \rightarrow 0$, then its asymptotic behavior [see Eq. (15)]

$\psi(M / r \rightarrow 0) \rightarrow 0$

would immediately imply that it must possess (at least) one extremum point $r_{\text {peak }} \in(0, \infty)$ in the curved spacetime (1). It therefore remains to be proved that nonminimally coupled massive scalar fields which are characterized by the nearorigin behavior

$\psi(r \rightarrow 0) \neq 0$

must also posses an extremum point.

For later purposes, we note that one finds from the energymomentum components (10) and (12) the simple functional relation

$T_{t}^{t}-T_{\phi}^{\phi}=e^{-\lambda} \frac{\xi\left(2 / r-v^{\prime}\right) \psi \psi^{\prime}}{1-8 \pi \xi \psi^{2}}$.

In particular, taking cognizance of Eqs. (2), (3), (13), and (18), one deduces the characteristic near-origin functional behavior

$\left|\frac{1}{r} \psi \psi^{\prime}\right|<\infty$ for $r \rightarrow 0$

for spatially regular scalar matter configurations. Likewise, taking cognizance of Eqs. (2), (3), (13), (17), (19), and the relation [see Eqs. (10) and (11)]

$$
T_{t}^{t}-T_{r}^{r}=e^{-\lambda} \frac{(2 \xi-1)\left(\psi^{\prime}\right)^{2}+2 \xi \psi \psi^{\prime \prime}-\xi(\nu+\lambda)^{\prime} \psi \psi^{\prime}}{1-8 \pi \xi \psi^{2}},
$$

one finds the bounded near-origin functional behavior

$\left|\psi^{\prime \prime}\right|<\infty$ for $r \rightarrow 0$

for spatially regular static field configurations.

Substituting the Ricci scalar curvature ${ }^{8}$

$$
\begin{aligned}
R= & -\frac{8 \pi}{1-8 \pi \xi \psi^{2}}\left\{e ^ { - \lambda } \left[\xi\left(\frac{12}{r}+3 v^{\prime}-3 \lambda^{\prime}\right) \psi \psi^{\prime}\right.\right. \\
& \left.\left.+6 \xi \psi \psi^{\prime \prime}+(6 \xi-1)\left(\psi^{\prime}\right)^{2}\right]-2 \mu^{2} \psi^{2}\right\}
\end{aligned}
$$

which characterizes the spherically symmetric self-gravitating massive scalar fields into Eq. (9), one obtains the ordinary differential equation

$$
\begin{aligned}
\mathcal{F} & \cdot \psi^{\prime \prime}+\psi^{\prime} \cdot\left[\frac{1}{2}\left(\frac{4}{r}+v^{\prime}-\lambda^{\prime}\right) \mathcal{F}+8 \pi \xi(6 \xi-1) \psi \psi^{\prime}\right] \\
& -\mu^{2} e^{\lambda}\left(1+8 \pi \xi \psi^{2}\right) \psi=0,
\end{aligned}
$$

which determines the radial behavior of the nonminimally coupled scalar matter configurations, where

$\mathcal{F}(r ; \xi) \equiv 1+8 \pi \xi(6 \xi-1) \psi^{2}$.

For later purposes we note that, taking cognizance of Eq. (16), one finds that $\mathcal{F}(r ; \xi)$ is characterized by the simple asymptotic behavior

$\mathcal{F}(M / r \rightarrow 0) \rightarrow 1$.

The radially-dependent function $\mathcal{F}(r ; \xi)$ is obviously positive definite for nonminimally coupled massive scalar fields in the dimensionless regimes $\xi \geq 1 / 6$ and $\xi \leq 0$ [see Eq. (24)]. We shall now prove that this property also characterizes the function $\mathcal{F}(r)$ in the complementary dimensionless regime $0<\xi<1 / 6$ of the nonminimal coupling parameter. Let us assume that the function $\mathcal{F}(r)$ has a root at some point $r_{0} \in(0, \infty)$, in which case one finds from (23) the functional relation ${ }^{9}$

$8 \pi \xi(6 \xi-1)\left(\psi^{\prime}\right)^{2}=\mu^{2} e^{\lambda}\left(1+8 \pi \xi \psi^{2}\right) \quad$ at $\quad r=r_{0}$.

Taking cognizance of the fact that, in the dimensionless regime $0<\xi<1 / 6$, the 1.h.s of (26) is non-positive whereas the r.h.s of (26) is positive definite, one deduces that the equality sign in (23) cannot be respected at the assumed $\operatorname{root} r=r_{0}$ of $\mathcal{F}(r)$. We therefore learn that the radial function $\mathcal{F}(r)$ has no roots. In particular, using the characteristic asymptotic

\footnotetext{
8 Here we have used the expressions (10), (11), and (12) for the components of the energy-momentum tensor together with the Einstein relation $R=-8 \pi T$ between the Ricci scalar curvature and the trace of the corresponding energy-momentum tensor.

${ }^{9}$ Note that one finds from Eq. (24) the trivial relation $\psi\left(r=r_{0}\right) \neq 0$ at the assumed root of $\mathcal{F}$.
} 
relation (25), one finds that the radially-dependent function $\mathcal{F}(r ; \xi)$ is characterized by the simple inequality

$\mathcal{F}(r)>0 \quad$ for $\quad r \in(0, \infty)$

in the entire curved spacetime.

Taking cognizance of Eqs. (2), (3), (19), (21), and (23), one obtains the near-origin $(r \rightarrow 0)$ radial equation

$\psi^{\prime \prime}+\frac{2}{r} \psi^{\prime}-\mu^{2} \mathcal{F}^{-1}\left(1+8 \pi \xi \psi^{2}\right) \psi=0$,

whose physically acceptable solution ${ }^{10}$ is characterized by the small-r spatial behavior ${ }^{11,12}$

$\psi(r \rightarrow 0)=B\left[1+\frac{1}{6} \mu^{2} \frac{1+8 \pi \xi B^{2}}{1+8 \pi \xi(6 \xi-1) B^{2}} \cdot r^{2}\right]+O\left(r^{3}\right)$,

where $B$ is a constant. Interestingly, and most importantly for our analysis, one learns from Eqs. (24), (27), and (29) that spatially regular nonminimally coupled massive scalar fields with $\xi \geq 0$ are characterized by the near-origin functional relations

$\psi \psi^{\prime}(r \rightarrow 0)=0 ; \psi \psi^{\prime \prime}(r \rightarrow 0)>0$.

The asymptotic large- $r$ and small- $r$ functional behaviors (16) and (30), when combined together, reveal the important fact that the characteristic radial eigenfunction $\psi(r)$ of the spatially regular nonminimally coupled massive scalar fields must possess, in accord with our previous assertion, (at least) one extremum point $r_{\text {peak }} \in(0, \infty)$ in the curved spacetime (1). In particular, at this extremum point the scalar eigenfunction is characterized by the functional relations

$\left\{\psi \neq 0 ; \psi^{\prime}=0 ; \psi \cdot \psi^{\prime \prime}<0\right\}$ for $r=r_{\text {peak }}$.

Substituting (31) into the radial differential equation (23), one finds

$\mathcal{F} \cdot \psi \psi^{\prime \prime}=\mu^{2} e^{\lambda}\left(1+8 \pi \xi \psi^{2}\right) \psi^{2} \quad$ at $\quad r=r_{\text {peak }}$.

Interestingly, taking cognizance of the analytically derived relations (27) and (31) one learns that, in the dimensionless physical regime $\xi \geq 0$, the 1.h.s of (32) is negative definite whereas the r.h.s of (32) is positive definite. Thus, for

\footnotetext{
10 That is, the mathematical solution of the radial differential equation (28) which respects the characteristic near-origin functional relations (19) and (21) for spatially regular scalar eigenfunctions.

11 It is worth emphasizing that one learns from Eqs. (17), (19), (21), and (29) that $\mathcal{F}(r)$ cannot vanish in the near-origin $r \rightarrow 0$ limit.

12 Note that $B \equiv \psi(r \rightarrow 0) \neq 0$ [see Eqs. (17) and (29)]. As discussed above, if the scalar eigenfunction is characterized by the nearorigin relation $\psi(r \rightarrow 0)=0$, then the asymptotic radial behavior (16) implies, in accord with the above stated assertion, that $\psi(r)$ is a non-monotonic function.
}

spatially regular static scalar configurations, the radial differential equation (23) cannot be satisfied at the characteristic extremum point (31).

We therefore conclude that static spatially regular massive scalar fields whose nonminimal coupling parameter lies in the dimensionless physical regime $\xi \geq 0$ cannot form spherically symmetric asymptotically flat horizonless matter configurations (boson stars).

\section{Summary}

Boson stars represent self-gravitating horizonless compact objects which are made of massive scalar fields. The physical and mathematical properties of these spatially regular bound-state matter configurations have attracted the attention of physicists and mathematicians during the last three decades (see [1,2] and references therein).

In the present paper we have presented a novel nonexistence theorem for static boson stars. In particular, the static sector of the non-linearly coupled Einstein-matter field equations has been studied analytically for spatially regular self-gravitating massive scalar fields with nonminimal coupling to gravity. Interestingly, our compact no-go theorem explicitly rules out the existence of asymptotically flat horizonless spacetimes that contain spatially regular boson stars which are made of nonminimally coupled ${ }^{13}$ massive scalar fields.

Acknowledgements This research is supported by the Carmel Science Foundation. I would like to thank Yael Oren, Arbel M. Ongo, Ayelet B. Lata, and Alona B. Tea for helpful discussions.

Data Availability Statement This manuscript has no associated data or the data will not be deposited. [Authors' comment: I would like to emphasize that all relevant physical and mathematical calculations are explicitly presented in this paper.]

Open Access This article is distributed under the terms of the Creative Commons Attribution 4.0 International License (http://creativecomm ons.org/licenses/by/4.0/), which permits unrestricted use, distribution, and reproduction in any medium, provided you give appropriate credit to the original author(s) and the source, provide a link to the Creative Commons license, and indicate if changes were made. Funded by $\mathrm{SCOAP}^{3}$.

\footnotetext{
${ }^{13}$ It is worth noting again that our compact no-go theorem is valid for non-negative $\xi \geq 0$ values of the dimensionless physical parameter $\xi$ which quantifies the coupling between the massive scalar field and the characteristic curvature of the spherically symmetric static spacetime [see Eq. (5)]. As emphasized above, the non-existence of spatially regular static boson stars made of nonminimally coupled massive scalar fields in the complementary regime $\xi<0$ of the dimensionless nonminimal coupling parameter $\xi$ can be deduced from the large- $r$ asymptotic analysis presented in [13].
} 


\section{References}

1. F.E. Schunck, E.W. Mielke, Class. Quantum Gravity 20, R301 (2003)

2. S.L. Liebling, C. Palenzuela, Living Rev. Relat. 15, 6 (2012)

3. J.E. Chase, Commun. Math. Phys. 19, 276 (1970)

4. J.D. Bekenstein, Phys. Rev. Lett. 28, 452 (1972)

5. C. Teitelboim, Lett. Nuov. Cim. 3, 326 (1972)

6. M. Heusler, J. Math. Phys. 33, 3497 (1992)

7. M. Heusler, Class. Quant. Grav. 12, 779 (1995)

8. J.D. Bekenstein, Phys. Rev. D 51, R6608 (1995)

9. A.E. Mayo, J.D. Bekenstein, Phys. Rev. D 54, 5059 (1996)

10. J. D. Bekenstein, arXiv:gr-qc/9605059

11. S. Hod, Phys. Rev. D 94, 104073 (2016). arXiv: 1612.04823

12. S. Hod, Phys. Rev. D 96, 024019 (2017). arXiv: 1709.01933

13. S. Hod, Phys. Lett. B 771, 521 (2017)

14. S. Hod, Phys. Lett. B 778, 239 (2018)

15. S. Hod, Phys. Rev. D 86, 104026 (2012). arXiv: 1211.3202

16. S. Hod, Eur. Phys. J. C 73, 2378 (2013). arXiv: 1311.5298

17. S. Hod, Phys. Rev. D 90, 024051 (2014). arXiv: 1406.1179

18. S. Hod, Phys. Lett. B 739, 196 (2014). arXiv:1411.2609

19. S. Hod, Class. Quantum Gravity 32, 134002 (2015). arXiv: 1607.00003

20. S. Hod, Phys. Lett. B 751, 177 (2015)

21. S. Hod, Class. Quantum Gravity 33, 114001 (2016)

22. S. Hod, Phys. Lett. B 758, 181 (2016). arXiv:1606.02306

23. S. Hod, O. Hod, Rapid communication. Phys. Rev. D 81, 061502 (2010). arXiv:0910.0734

24. S. Hod, Phys. Lett. B 708, 320 (2012). arXiv:1205.1872

25. S. Hod, J. High Energy Phys. 01, 030 (2017). arXiv:1612.00014

26. C.A.R. Herdeiro, E. Radu, Phys. Rev. Lett. 112, 221101 (2014)
27. C.L. Benone, L.C.B. Crispino, C. Herdeiro, E. Radu, Phys. Rev. D 90, 104024 (2014)

28. C.A.R. Herdeiro, E. Radu, Phys. Rev. D 89, 124018 (2014)

29. C.A.R. Herdeiro, E. Radu, Int. J. Mod. Phys. D 23, 1442014 (2014)

30. Y. Brihaye, C. Herdeiro, E. Radu, Phys. Lett. B 739, 1 (2014)

31. J.C. Degollado, C.A.R. Herdeiro, Phys. Rev. D 90, 065019 (2014)

32. C. Herdeiro, E. Radu, H. Rúnarsson, Phys. Lett. B 739, 302 (2014)

33. C. Herdeiro, E. Radu, Class. Quantum Gravity 32, 144001 (2015)

34. C.A.R. Herdeiro, E. Radu, Int. J. Mod. Phys. D 24, 1542014 (2015)

35. C.A.R. Herdeiro, E. Radu, Int. J. Mod. Phys. D 24, 1544022 (2015)

36. P.V.P. Cunha, C.A.R. Herdeiro, E. Radu, H.F. Rúnarsson, Phys. Rev. Lett. 115, 211102 (2015)

37. B. Kleihaus, J. Kunz, S. Yazadjiev, Phys. Lett. B 744, 406 (2015)

38. C.A.R. Herdeiro, E. Radu, H.F. Rúnarsson, Phys. Rev. D 92, 084059 (2015)

39. C. Herdeiro, J. Kunz, E. Radu, B. Subagyo, Phys. Lett. B 748, 30 (2015)

40. C.A.R. Herdeiro, E. Radu, H.F. Rúnarsson, Class. Quantum Gravity 33, 154001 (2016)

41. C.A.R. Herdeiro, E. Radu, H.F. Rúnarsson, Int. J. Mod. Phys. D 25, 1641014 (2016)

42. Y. Brihaye, C. Herdeiro, E. Radu, Phys. Lett. B 760, 279 (2016)

43. Y. Ni, M. Zhou, A.C. Avendano, C. Bambi, C.A.R. Herdeiro, E. Radu, JCAP 1607, 049 (2016)

44. M. Wang, arXiv: 1606.00811

45. S. Hod, Phys. Lett. B 773, 208 (2017)

46. S. Hod, Phys. Rev. D 84, 124030 (2011). arXiv: 1112.3286

47. S. Hod, Phys. Rev. D 84, 104024 (2011). arXiv:1201.0068

48. S. Hod, Phys Lett. B 727, 345 (2013). arXiv:1701.06587 